\title{
BMJ Open Canadian clinical practice guidelines for the use of plant-based cannabis and cannabinoid-based products in the management of chronic non-cancer pain and co-occurring conditions: protocol for a systematic literature review
}

Patrick Wright, ${ }^{1}$ Zach Walsh, ${ }^{2}$ Shari Margolese, ${ }^{3}$ Tatiana Sanchez, ${ }^{2}$ Stephanie Arlt, ${ }^{4}$ Lynne Belle-Isle, ${ }^{1}$ Michelle St.Pierre, ${ }^{2}$ Alan Bell, ${ }^{5}$ Paul Daeninck, ${ }^{6}$ Marilou Gagnon, ${ }^{7}$ Gary Lacasse, ${ }^{1}$ Caroline MacCallum, ${ }^{8}$ Enrico Mandarino, ${ }^{9,10}$ Janet Yale, ${ }^{11}$ James O'Hara, ${ }^{12}$ Cecilia Costiniuk (i) ${ }^{13}$

To cite: Wright $\mathrm{P}$, Walsh $\mathrm{Z}$, Margolese S, et al. Canadian clinical practice guidelines for the use of plant-based cannabis and cannabinoid-based products in the management of chronic non-cancer pain and co-occurring conditions: protocol for a systematic literature review. BMJ Open 2020;10:e036114. doi:10.1136/ bmjopen-2019-036114

- Prepublication history and additional material for this paper are available online. To view these files, please visit the journal online (http://dx.doi. org/10.1136/bmjopen-2019036114).

Received 02 December 2019 Revised 25 March 2020 Accepted 07 April 2020

Check for updates

(C) Author(s) (or their employer(s)) 2020. Re-use permitted under CC BY-NC. No commercial re-use. See rights and permissions. Published by BMJ.

For numbered affiliations see end of article.

Correspondence to

Mr Gary Lacasse;

gary.lacasse@cdnaids.ca

\section{ABSTRACT}

Introduction Chronic pain and co-occurring disorders, such as sleep disorders, anxiety, depression, posttraumatic stress disorder and substance use disorders, are among the most common conditions for which cannabis and cannabinoid-based products derived from the cannabis plant (CBP) are used for therapeutic purposes. However, healthcare providers report that they lack sufficient information on the risks, benefits and appropriate use of cannabis and CBP derived from the cannabis plant for therapeutic purposes.

Methods and analysis We will conduct a systematic review of studies investigating the use of cannabis and CBP derived from the cannabis plant for the treatment of chronic pain and co-occurring conditions. Randomised controlled trials, meta-analyses and observational studies will be prioritised. We will exclude reviews of cannabinoid mechanisms of actions, commentary articles and narrative reviews. The primary outcome of interest will be efficacy in relieving chronic pain. Secondary outcomes will be efficacy in ameliorating conditions such as sleep disorders, anxiety, depression, post-traumatic stress disorder and substance use disorders. We will search electronic bibliographic databases including Academic Search Complete, Cochrane Database of Systematic Reviews, Evidence based Medicine Reviewes, OVID Medline, PsychINF0, PubMed, CINAHL and Web of Science. Two reviewers will conduct screening and data collection independently. Study level of bias will be assessed using the Cochrane Risk of Bias Assessment Tool for randomised controlled trials and non-randomised studies. Narrative analysis will be utilised to interpret the data.

Ethics and dissemination The results of this systematic review will inform guideline development for the use of cannabis and CBP derived from the cannabis plant in the management of chronic pain and co-occurring conditions. Areas requiring further study will also be highlighted. PROSPERO registration number CRD42020135886.
Strengths and limitations of this study

- Extensive review of literature with rigorous study selection and methods for data extraction, quality assessment and data synthesis.

- Breadth and consideration of diverse methodologies distinguishes this review for other recent reviews of cannabis and pain.

- Wide variety of panel members comprised of clinicians, academics and community members with unique perspectives and synergistic skills.

- A timely systematic review given liberalisation of cannabis regulations across Europe and the Americas.

- Conclusions may be limited by inclusion of relatively few controlled trials.

\section{BACKGROUND}

Approximately 19\%-29\% of Canadian adults aged 18 and older live with chronic pain, most commonly attributed to lower back pain and arthritis, with an average duration of more than 10 years. ${ }^{12}$ Arthritis alone, which includes more than 100 rheumatic diseases and conditions that affect joints, affects over 4.2 million Canadians (16\% of those aged 15 years and older), and this prevalence is estimated to reach approximately 7 million, or 1 in 5 Canadians aged 15 and older, by $2031 .^{3}$

Chronic pain often co-occurs with sleep disorders, anxiety, depression, post-traumatic stress disorder and substance use disorders such as opioid use disorder and alcohol use disorder. ${ }^{4-10}$ Chronic pain and these co-occurring conditions are also among the most common conditions for which 
cannabinoid-based products derived from the cannabis plant (CBP) are used for therapeutic purposes. ${ }^{11-14}$

The cannabis plant contains over 100 phytocannabinoids, although $\Delta^{9}$-tetrahydrocannabinol and cannabidiol are the most well-characterised. Other cannabinoids contained in the plant include cannabigerol, cannabichromene, cannabinodiol, cannabielsoin, cannabicyclol, cannabinol, cannabitriol and others. ${ }^{15} 16$ The cannabis plant also contains terpenoids which provide characteristic aromas. ${ }^{17}$ Different cannabinoids and terpeneoids in combination behave in synergy, through what has been coined 'the entourage effect', explaining why plants are often more efficacious than their components in isolation. ${ }^{18}$ Extracts include nabiximols (Sativex), a 1:1 tetrahydrocannabinol (THC):cannabidiol (CBD) sublingual spray. Synthetic pharmaceutical-grade cannabinoids include nabilone (Cesamet) and dronabinol (Marinol),synthetic products administered orally by capsule. A major knowledge gap relates to the use of cannabis and plant-derived cannabinoidsderived from the cannabis plant in the management of chronic pain and co-occurring conditions.

In Canada, surveys indicate that patients frequently treat multiple symptoms with CBP derived from the cannabis plant. ${ }^{14}$ Since 2001, Canada has had a federal programme that authorises the use of CBP derived from the cannabis plant and as of October 2018, has legalised and regulated the sale of cannabis for adult recreational use. Thus, for Canadians, the role of CBP derived from the cannabis plant in the context ofchronic pain management and its associated co-occurring conditions is likely to increase. Managing chronic pain and co-occurring morbidities is a complex public health and medical challenge, which is compounded by the introduction of CBP into the pharmacopoeia of therapeutic options.

Healthcare providers have expressed concerns about the use of CBP derived from the cannabis plant, stating that they did not have the quality of evidence they require to feel comfortable discussing CBP derived from the cannabis plant as a therapeutic option with their patients. ${ }^{19}$ They reported that they lack sufficient information on risks, benefits and appropriate use of CBP derived from the cannabis plant for therapeutic purposes and were reluctant to support their patients' request for access to CBP. ${ }^{2021}$

The frequent co-occurrence of chronic pain and substance use disorders is often explained as patients' self-medicating to manage living with chronic pain. ${ }^{6}$ Approximately $21 \%-29 \%$ of individuals prescribed opioids for chronic pain misuse them, while 8\%-12\% develop opioid use disorder ${ }^{22-26}$ CBPsubstitution for opioids is increasingly reported in the literature. ${ }^{27-30}$ The potential for CBP use as a drug-related harm reduction strategy is being recognised ${ }^{29} 3132$; however, it is not without risks, as its use may be associated with an increased risk of relapse. ${ }^{33-35}$ Regardless of the hypothesis that links chronic pain and substance use disorders, understanding the role of CBPd erived from the cannabis plant in this context is crucial for the development of clinical practice guidelines.

Recent years have seen a proliferation of systematic literature reviews on CBP and their effects on chronic pain and co-occurring conditions. Systematic reviews have been conducted on CBP and chronic pain ${ }^{36-39}$; sleep disorders ${ }^{39}{ }^{40}$ and mood disorders. ${ }^{3941}$ While a few publications offer recommendations regarding administration and dosing strategies ${ }^{42}{ }^{43}$ and one recent publication offers clinical practice guidelines for prescribing CBP in primary care, ${ }^{44}$ clinicians and patients have no specific guidance on the use of CBP for the management of chronic pain and co-occurring conditions. Given the new legal regimes globally and in Canada regarding recreational cannabis and CBP derived from the cannabis plant, healthcare providers need to be aware of the efficacy of CBP derived from the cannabis plant in regards to chronic pain and confident in knowing when such therapies may be beneficial for their patients.

There is a need for detailed, up-to-date tools and information for healthcare providers and patients to assist them with decisions about CBP derived from the cannabis plant as a treatment option. We propose to develop the Canadian Clinical Practice Guidelines for the Use of Cannabis and Cannabinoid-Based Products in the Management of Chronic Pain and Co-Occurring Conditions. Of note, to fill an important knowledge gap, these guidelines will examine literature focused on cannabis and CBP derived from the cannabis plant rather than synthetic, pharmaceutical-grade cannabinoids.

\section{METHODS AND ANALYSIS \\ Outcome(s) \\ Primary outcome: chronic pain}

Chronic pain includes any painful condition that persists for more than 3 months, including nociceptive, neuropathic and centralised pain. ${ }^{13}{ }^{45}$ Chronic pain outcomes are measured with scales, including but not limited to: the numeric rating scale, a visual analogue scale, EuroQuality of life-5 Dimensions-5 Levels (EQ-5D5L), Profile of Mood States (POMS) Questionnaire, 36-item shortform survey (FS36), the Neuropathic Pain Scale and the McGill Pain Questionnaire. ${ }^{37}$ Some of these examples importantly include measurements that focus on patient reported outcomes, patient functionality and quality of life.

\section{Secondary outcomes \\ Sleep disorders}

Although many sleep disorders exist, insomnia is the most common. Insomnia refers to a condition whereby sleep is disturbed despite the presence of an adequate opportunity and circumstance for sleep, which has a negative effect on daily function. ${ }^{46}$ Sleep measures include sleep behaviour inventory, sleep evaluation questionnaire, electro-encephalogram (EEG) measures and visual observation of sleep activity. ${ }^{40}$ 
Anxiety, depression and post-traumatic stress disorder

The co-occurrence of chronic pain and mood disorders such as anxiety, depression and post-traumatic stress disorder is well documented. ${ }^{45710}$ Mood disorder outcomes are measured by Structured Clinical Interview for Diagnostic and Statistical Manual of Mental Disorders (DSM) and self-reported questionnaire (eg, self report, with the Beck Depression Inventory, Hamilton Depression Inventory, Centre for Epidemiological Studies Depression Scale (CES-D)).

\section{Substance use disorders}

Changes in the use of non-cannabinoid products and other substances, in conjunction with cannabis use, will be reviewed. CBP derived from the cannabis plant substitution is assessed through questionnaires. DSM-V diagnoses for drug abuse and dependence can be obtained using instruments such as the WHO Composite International Diagnostic Interview (CIDI), Drug User Disorder Identification Test (DUDIT), Alcohol, Smoking, and Substance Involvement Screening Test (ASSIST) and others. ${ }^{6} 47$ Alcohol use disorder and opioid use disorder are also often measured using specific instruments including The Alcohol Use Disorders Identification Test (AUDIT), ${ }^{48}$ the Alcohol Use Disorder and Associated Disabilities Interview Schedule-IV (AUDADIS-IV) ${ }^{49}$ and the Current Opioid Misuse Measure (COMM), ${ }^{50}$ as well as others.

Previous studies have focused on risks and harms associated with cannabis and few have addressed the health promoting or beneficial effects of CBP derived from the cannabis plant. $^{51}{ }^{52}$ However, as the development of a cannabis use disorder is a possible consequence of cannabis consumption in susceptible individuals, the presence of cannabis use disorders will be noted. Specific screening and diagnostic instruments to assess cannabis use disorders include the Cannabis Problems Questionnaire (CPQ), Cannabis Abuse Screening Test (CAST), Cannabis Use Disorder Identification Test (CUDIT) and its revised version (CUDIT-R) and others. ${ }^{47} 53$

\section{Search strategy}

An electronic search will be conducted for peer-reviewed articles (2001-2019), restricted to the English language, in the following electronic bibliographic databases: Academic Search Complete, Cochrane Database of Systematic Reviews (CDSR), Evidence Based Medicine Reviews (EBMR), OVID Medline, PsychINFO, PubMed, CINAHL and Web of Science. The search strategy will include the following controlled vocabulary and relevant key terms:

(cannabi* OR marijuana OR endocannabi* OR THC OR Tetrahydrocannabinol OR weed OR CBD OR Indica OR Sativa ORnabiximols OR dronabinol OR pot) AND (pain OR headache OR neuralgia OR migraine)

This search strategy was developed with the assistance of a medical librarian experienced is systematic reviews. As the journal Cannabis and Cannabinoid Research is currently one of the few journals specifically devoted to cannabis research, this journal will be hand searched for studies that meet the inclusion criteria. Based on the recommendations of the medical librarian, the terms 'nabiximols' and 'dronabinol' were included in the search strategy to ensure that we capture all relevant studies to screen. However, studies focused exclusively on the efficacy of synthetic cannabinoids of pharmaceutical grade (such as nabilone or dronabinol) approved for human use will be excluded. As nabiximols contain plant derived cannabinoids, they will be included. Only studies published since 2001 will be included to focus the review on recent evidence. Since 2001 there have been technological advances and regulatory changes, such as the legalisation of medicinal cannabis in Canada, that may have improved the quality of research. All database searches will be completed by May 2019.

\section{Study screening and inclusion}

Following the implementation of our search, we will obtain the titles and abstracts from all references. First we will examine the tiles and abstracts, and then fulltexts of studies which appear relevant will be screened by two reviewers independently. We will conduct pilot exercises to identify and address any inconsistencies in applying the screening criteria. The inclusion and exclusion criteria for each stage of screening are indicated below. When no abstract is available, and the article cannot be confidently excluded by solely the title, the full-text will be obtained. In general, if there is uncertainty as to whether a study should be excluded, the study will proceed to the full-text screen. Two reviewers will resolve disagreements on inclusion, and a third person will reconcile any remaining disagreements. We will not exlude studies based on poor research quality, but we will note the low quality. The process of study selection will be summarised using a Preferred Reporting Items for Systematic Reviews and Meta-Analyses (PRISMA) flow diagram. ${ }^{54}$

\section{Study eligibility criteria}

Study selection will be based on the criteria listed in table 1. Study inclusion and exclusion criteria are listed in table 2 .

\section{Data extraction}

Selection of studies

Using the PRISMA conventions, ${ }^{55}$ an Evidence Synthesis Working Group, working with the Guidelines Panel, will determine eligibility of studies by reading the abstracts identified by the search. Grey literature will also be included when appropriate. Studies will be selected based on inclusion and exclusion criteria. The Evidence Synthesis Working Group will independently read the selected studies and reach agreement about inclusion and exclusion by discussion. A PRISMA flow chart will be created. The CBP Task Force will come to the final conclusion when there is debate. 


\begin{tabular}{ll} 
Table 1 PICOS breakdown of study eligibility criteria \\
\hline Category & Description of criteria \\
\hline Population & $\begin{array}{l}\text { Human of any age living with chronic, or non-acute, pain (pain of greater than } 3 \text { month duration) } \\
\text { Humans of any age living with chronic pain and co-occurring conditions: sleep disorders, mood disorders } \\
\text { (anxiety, depression, post-traumatic stress disorder), alcohol use disorder and opioid use disorder }\end{array}$ \\
Intervention & $\begin{array}{l}\text { Cannabis or cannabinoid-based products (CBP) derived from the cannabis plant in the form of herbal } \\
\text { cannabis and derivatives }\end{array}$
\end{tabular}

$\begin{array}{ll}\text { Comparison(s) } & \text { Placebo or other medications or intervention } \\ & \text { Studies without comparators will also be included* }\end{array}$

Outcome(s) Primary outcome

(1) Efficacy, tolerability and safety of cannabis and CBP derived from the cannabis plant in the management of chronic pain.

(2) Improvement in chronic pain, symptom management.

(3) Improvement in quality of life, patient-reported outcomes and patient functionality.

Secondary outcomes

Improvement in sleep disorders, anxiety, depression, alcohol use disorder, and opioid use disorder

$\begin{array}{ll}\text { Study design } & \text { Randomised controlled trials, controlled trials,studies listed in meta-analyses and observational studies will } \\ \text { be included }\end{array}$

Studies that focus on cannabinoid mechanisms, commentary articles or non-systematic reviews will be excluded

*An example of a study without a comparator would be a study examining the efficacy of a single dosing regimen comparing baseline to end study scores.

PICOS, Patient, Intervention, Comparison intervention, Outcome, Study design.

\section{Data extraction and management}

The Evidence Synthesis Working Group will extract data from the selected studies independently using a standardised Data Extraction Form (online supplementary file 1) to create evidence tables. For each study, relevant data will be extracted related to study identification (author, year published, number and location of centres, funding, journal name), the number of participants, form of CBP derived from the cannabis plant, dose and route, study design and setting, inclusion and exclusion criteria of the study sample, aggregate demographic (age, sex, type of pain, co-occurring conditions) and clinical characteristics (co-morbidities), and outcome measures (eg, scores on the Visual Analogue Scales or McGill Pain Questionnaire) and results.We will also record adverse events as reported in individual studies, including the frequency and severity of cases when applicable. Adverse events will collectively be analysed using the WHO Toxicity grading scale for determining the safety of adverse events.

In addition, we plan to examine secondary outcomes within standalone studies on the use of cannabis (eg, effects of cannabinoids on anxiety) as well as within studies of cannabinoids being used to manage chronic pain (eg, looking at anxiety as a secondary outcome in a pain clinical trial). Records of all searches will be kept on secure databases only accessible to the investigators. Records of all data extraction forms and consensus discussions will also be kept on the same databases.

\section{Strategy for data synthesis}

Data will be extracted from reviews, including existing meta-analyses, using a standardised data extraction tool. Due to the high variability in previous cannabis research, a meta-analysis is likely inappropriate. This variability is due to heterogeneity of sample populations, study types and lengths, and CBP derived from the cannabis plant interventions (eg, CBP type, dosing, administration route, etc). Similar challenges have prevented the execution of meta-analyses in previous, related reviews. ${ }^{56}$ Patterns related to efficacy, safety, tolerability will be explored through narrative synthesis. ${ }^{567}$ Data from relevant categories (eg, subpopulations, age groups, alternative therapies, etc) will be compiled based on the availability of quality evidence. Consistent findings and discrepancies will be discussed. Findings will be aggregated or synthesised to generate a set of statements rated according to their quality. We do not plan to conduct a meta-analysis.

\section{Assessment of evidence and recommendations}

The Task Force will use the Grading of Recommendations Assessment, Development and Evaluation GRADE) system to rate the quality of the evidence and strength of its recommendations. ${ }^{58-64}$

\section{Analysis of subgroups or subsets}

Evidence for the use of CBP derived from the cannabis plant in the management of chronic pain and co-occurring conditions will be presented for clinical considerations related to efficacy, tolerability, safety, indications, 
Table 2 Inclusion and exclusion criteria

Inclusion Cannabis and the management of chronic pain.

criteria Cannabis and the management of chronic pain and co-occurring conditions: sleep disorders, mood disorders (anxiety, depression, post-traumatic stress disorder), alcohol use disorder and opioid use disorder.

- Efficacy, tolerability and safety studies on the use of cannabis in the management of chronic pain.

- Indications and dosing strategies of cannabis for the treatment of chronic pain.

- Drug interactions, adverse events, negative effects and contraindications for the use of cannabis in the treatment of chronic pain.

- Considerations regarding the use of cannabis for the management of chronic pain for individuals with a history of sleep disorders, anxiety, depression, post-traumatic stress disorder, opioid use disorder and alcohol use disorder.

- The substitution effect of cannabis for medications or other drugs in the context of the management of chronic pain.

$\begin{array}{ll}\begin{array}{l}\text { Exclusion } \\ \text { criteria }\end{array} & \text { Studies published before } 2001 . \\ & \text { Studies in a language other than English. } \\ & \text { recreational vs medicinal use. } \\ & \text { Studies focused exclusively on synthetic cannabinoids of pharmaceutical grade approved for human use. } \\ & \text { Studies focused on the prevention or cessation of cannabis use. } \\ & \text { Studies focused exclusively on cancer-related pain. } \dagger \\ & \text { Studies focused on cannabis use disorder. } \\ & \text { Studies where cannabis is only one aspect of an intervention, and not the main focus. } \\ & \text { Studies on non-humans/animals. }\end{array}$

*These compounds should be distinguished from those used in basic science research, not approved for human use, and which are known on the streets by terms such as 'Spice' and 'K2'.

†Due to the large number of studies focused exclusively on cancer-related pain, we have excluded these studies from the current systematic review in order to narrow the focus. However, we acknowleddge the importance of cancer-related pain and suggest that this be the focus of a separate systematic review.

dosing, drug interactions, adverse events, negative effects and contraindications. Evidence regarding considerations related to the use of CBP derived from the cannabis plantfor patients with a history of substance use disorder. The phenomenon of CBP substitution for other drugs will be included.

\section{Risk of bias assessment}

Two reviewers (MSP and PW) will assess the potential bias and discrepancies will be discussed and adjudicated by the Data Synthesis committee (CC, ZW, SM). The National Institutes of Health risk of bias assessment tools ${ }^{65}$ will be used to assess the quality of included studies. These tools have been developed specifically for different study design types, and therefore the heterogeneity of included study designs will not affect the ability to assess quality appropriately. Each included study will be dually and independently reviewed and disagreements will be solved through discussion. These tools used for quality assessment are "not intended to create a list that is simply tallied up to arrive at a summary judgement of quality', meaning reviewers will evaluate studies utilising the tools but will not solely rely on the cumulative score, and will make decisions through discussion when necessary. Studies will be graded as either 'good quality' (score of 3), implying low risk of bias, 'fair quality' (score of 2) implying some risk of bias or 'poor quality' (score of 1), implying high risk of bias. Assessment of bias will be performed at the overall study level. Specific Questions to assess for study limitations and the risk of bias are included on our Data Extraction Form (online supplementary file 1).

\section{Data analysis/synthesis}

Findings from the review will be synthesised to highlight where multiple reviews find consistent effects and where reviews have come to different conclusions about the strength of the evidence. In the narrative synthesis, we will discuss the findings both within and between studies, based on guidance from the Centre for Reviews and Dissemination (eg, a study examining the efficacy of a single dosing regimen comparing baseline to end study scores). Findings will be aggregated or synthesised to generate a set of statements rates according to their quality.

\section{Reporting of the review}

The Cannabis Guidelines Task Force plans on publishing both the protocol for the development of the clinical practice guidelines, as well as the systematic review protocol. Once the guidelines and decision aid are developed, they 
will also be published and disseminated. Members of the Task Force will be encouraged to present the guidelines at relevant conferences and meetings.

\section{Patient and public involvement}

Among the authors of this systematic review protocol are patient community advisors (SM and EM). They have been involved in all stages of this project, beginning from conception and design of this systematic review. They will continue to be involved at all stages, including study appraisal, guideline drafting and publication.

\section{DISCUSSION}

In this systematic review, we will prepare a detailed, up-to-date tool for healthcare providers and patients to assist them with decisions about CBP derived from the cannabis plantas a treatment option for chronic pain and co-occurring conditions including sleep disorders, mood disorders alcohol use disorder and opioid use disorder. Although some publications provide guidance with respect to administration and dosing of CBP derived from the cannabis plant ${ }^{42} 43$ and one recent publication offers clinical practice guidelines for prescribing CBP in primary care, ${ }^{44}$ our systematic review geared for both healthcare providers and patients will add to the current literature by providinga balanced view of both the benefits and potential risks, and will also highlight specific areas requiring additional research.

We anticipate some challenges with our systematic review. First, there is likely to be very high heterogeneity with regards to patient populations, CBP derived from the cannabis plantdosage form and dosages, study design and reported outcomes. When CBP is administered in different dosage forms, such as by capsule versus by inhaled form, the kinetics vary widely which may make direct, head-to-head comparisons between studies inappropriate. In addition, individual differnces in patient characteristics between studies may preclude us from generalising results across studies. Furthermore, our search terms may notenable us to pick up common chronic pain conditions such as arthritis, fibromyalgia, spinal cord injury and diabetic neuropathy. Nonetheless, within the context of chronic pain, our systematic review aims to provide a broad, balanced view of both the potential benefits and harms associated with the use of CBP for pain and co-occurring conditions. These results are likely to serve as an reference tool for both healthcare providers and patients suffering from such conditions, and will also underscore the specific areas of CBP research requiring further study.

\section{Study status}

At the time of protocol publication, discussions within the evidence synthesis working group have resulted in the plan to summarise data from systematic reviews separately from the data from original research. This data will be presented to the guidelines writing committee, who will draft the guidelines.

\section{Author affiliations}

${ }^{1}$ Canadian AIDS Society, Ottawa, Ontario, Canada

${ }^{2}$ Department of Psychology, University of British Columbia, Kelowna, British

Columbia, Canada

${ }^{3}$ Canadian HIV Trials Network Community Advisory Board, Vancouver, British Columbia, Canada

${ }^{4}$ Canadian Institute for Substance Use Research,University of Victoria, Victoria, British Columbia, Canada

${ }^{5}$ Department of Family and Community Medicine, University of Toronto, Toronto, Ontario, Canada

${ }^{6}$ Department of Internal Medicine, Max Rady College of Medicine, University of Manitoba, Winnipeg, Manitoba, Canada

${ }^{7}$ Canadian Institute for Substance Use Research, University of Victoria, Victoria, British Columbia, Canada

${ }^{8}$ Faculty of Medicine, University of British Columbia, Vancouver, British Columbia, Canada

${ }^{9}$ Community Advisory, Canadian Institutes of Health Research Canadian HIV Trials Network, Vancouver, British Columbia, Canada

${ }^{10} \mathrm{MJardin}$ Canada, Toronto, Ontario, Canada

${ }^{11}$ Arthritis Society of Canada, Toronto, Ontario, Canada

${ }^{12}$ Canadians for Fair Access to Medical Marijuana, Toronto, Ontario, Canada

${ }^{13}$ Chronic Viral IIIness Service, McGill University, Montreal, Quebec, Canada

\section{Twitter Michelle St.Pierre @ms_st_pierre}

Contributors PW and LB-I drafted the protocol. All authors, including MSP, AB, PD, $\mathrm{MG}, \mathrm{CM}, \mathrm{EM}, \mathrm{JY}$ and $\mathrm{JOH}$, had input into the protocol design and critically reviewed and revised the manuscript. TS and SA devised the search strategy, performed data extraction and data interpretation. PW and CC prepared the manuscript. The Data Synthesis Team (CC, ZW, SM, GL) was consulted for interpretation of the results. All authors conceived and designed the review, and read and approved the final manuscript. PW and CC are the guarantors of the review.

Funding Canadian Institutes for Health Research; Arthritis Society of Canada; Canopy Growth CorporationCC is supported by a Fonds de recherche du QuébecSanté Junior one career award.Neither the funders nor authors'affiliated institutions played any role in the development of the protocol.

Patient and public involvement Patients and/or the public were involved in the design, or conduct, or reporting, or dissemination plans of this research. Refer to the Methods section for further details.

Patient consent for publication Not required.

Provenance and peer review Not commissioned; externally peer reviewed.

Open access This is an open access article distributed in accordance with the Creative Commons Attribution Non Commercial (CC BY-NC 4.0) license, which permits others to distribute, remix, adapt, build upon this work non-commercially, and license their derivative works on different terms, provided the original work is properly cited, appropriate credit is given, any changes made indicated, and the use is non-commercial. See: http://creativecommons.org/licenses/by-nc/4.0/.

ORCID iD

Cecilia Costiniuk http://orcid.org/0000-0002-4547-714X

\section{REFERENCES}

1 Moulin DE, Clark AJ, Speechley M, et al. Chronic pain in Canada-prevalence, treatment, impact and the role of opioid analgesia. Pain Res Manag 2002;7:179-84.

2 Schopflocher D, Taenzer P, Jovey R. The prevalence of chronic pain in Canada. Pain Res Manag 2011;16:445-50.

3 Public Health Agency of Canada, Arthritis Consumer Experts ACREU, Canadian Arthritis Patients Alliance, Canadian Arthritis Network, Canadian Institute for Health Information, Statistics Canada \& The Arthritis Society. Life with Arthritis in Canada:A personal and public health challenge, 2010.

4 Asmundson GJG, Katz J. Understanding the co-occurrence of anxiety disorders and chronic pain: state-of-the-art. Depress Anxiety 2009;26:888-901.

5 Brennstuhl M-J, Tarquinio C, Montel S. Chronic pain and PTSD: evolving views on their comorbidity. Perspect Psychiatr Care 2015;51:295-304.

6 Ilgen MA, Perron B, Czyz EK, et al. The timing of onset of pain and substance use disorders. Am J Addict 2010;19:409-15. 
7 Lerman SF, Rudich Z, Brill S, et al. Longitudinal associations between depression, anxiety, pain, and pain-related disability in chronic pain patients. Psychosom Med 2015;77:333-41.

8 Morasco BJ, Gritzner S, Lewis L, et al. Systematic review of prevalence, correlates, and treatment outcomes for chronic noncancer pain in patients with comorbid substance use disorder. Pain 2011;152:488-97.

9 Russo EB, Guy GW, Robson PJ. Cannabis, pain, and sleep: lessons from therapeutic clinical trials of Sativex, a cannabis-based medicine. Chem Biodivers 2007;4:1729-43.

10 Yalcin I, Barrot M. The anxiodepressive comorbidity in chronic pain. Curr Opin Anaesthesiol 2014;27:520-7.

11 Belle-Isle L, Walsh Z, Callaway R, et al. Barriers to access for Canadians who use cannabis for therapeutic purposes. Int J Drug Policy 2014;25:691-9.

12 Belle-Isle L, Hathaway A. Barriers to access to medical cannabis for Canadians living with HIV/AIDS. AIDS Care 2007;19:500-6.

13 Hill KP, Palastro MD, Johnson B, et al. Cannabis and pain: a clinical review. Cannabis Cannabinoid Res 2017;2:96-104.

14 Walsh Z, Callaway R, Belle-Isle L, et al. Cannabis for therapeutic purposes: patient characteristics, access, and reasons for use. Int $J$ Drug Policy 2013;24:511-6.

15 Mechoulam R. Plant cannabinoids: a neglected pharmacological treasure trove. Br J Pharmacol 2005;146:913-5.

16 Elsohly M, Gul W. Consitutents of cannabis sativa. in Handbook of cannabis eRGPOUP, 2014.

17 Piomelli D, Russo EB. The Cannabis sativa Versus Cannabis indica Debate: An Interview with Ethan Russo, MD. Cannabis Cannabinoid Res 2016;1:44-6.

18 Mechoulam R, Ben-Shabat S. From gan-zi-gun-nu to anandamide and 2-arachidonoylglycerol: the ongoing story of cannabis. Nat Prod Rep 1999;16:131-43.

19 Jones C, Hathaway AD. Marijuana medicine and Canadian physicians: challenges to meaningful drug policy reform. In: Issues in Criminal SaRJ:Contemporary Justice Review. 2008: 11, 165-75.

20 Ziemianski D, Capler R, Tekanoff R, et al. Cannabis in medicine: a national educational needs assessment among Canadian physicians. BMC Med Educ 2015;15:52.

21 Survey Canadian Medical Assocation. Md role in use of medical marijuana baffles many doctors: survey, 2012.

22 Vowles KE, McEntee ML, Julnes PS, et al. Rates of opioid misuse, abuse, and addiction in chronic pain: a systematic review and data synthesis. Pain 2015;156:569-76.

23 Carlson RG, Nahhas RW, Martins SS, et al. Predictors of transition to heroin use among initially non-opioid dependent illicit pharmaceutical opioid users: a natural history study. Drug Alcohol Depend 2016;160:127-34.

24 Cicero TJ, Ellis MS, Surratt $\mathrm{HL}$, et al. The changing face of heroin use in the United States: a retrospective analysis of the past 50 years. JAMA Psychiatry 2014;71:821-6.

25 Speed TJ, Parekh V, Coe W, et al. Comorbid chronic pain and opioid use disorder: literature review and potential treatment innovations. Int Rev Psychiatry 2018:30:136-46.

26 Voon P, Karamouzian M, Kerr T. Chronic pain and opioid misuse: a review of reviews. Subst Abuse Treat Prev Policy 2017;12:36.

27 Boehnke KF, Litinas E, Clauw DJ. Medical cannabis use is associated with decreased opiate medication use in a retrospective cross-sectional survey of patients with chronic pain. $J$ Pain 2016;17:739-44.

28 Lucas P, Walsh Z, Crosby K, et al. Substituting cannabis for prescription drugs, alcohol and other substances among medical cannabis patients: the impact of contextual factors. Drug Alcohol Rev 2016;35:326-33.

29 Lucas P, Walsh Z. Medical cannabis access, use, and substitution for prescription opioids and other substances: a survey of authorized medical cannabis patients. Int J Drug Policy 2017;42:30-5.

30 Lucas P, Reiman A, Earleywine M, et al. Cannabis as a substitute for alcohol and other drugs: a dispensary-based survey of substitution effect in Canadian medical cannabis patients. Addict Res Theory 2013;21:435-42.

31 Lau N, Sales P, Averill S, et al. A safer alternative: cannabis substitution as harm reduction. Drug Alcohol Rev 2015;34:654-9.

32 Black N, Stockings E, Campbell G, et al. Cannabinoids for the treatment of mental disorders and symptoms of mental disorders: a systematic review and meta-analysis. Lancet Psychiatry 2019;6:995-1010.

33 Balhara YPS, Jain R. Cannabis use among opioid-dependent individuals on opioid substitution therapy. J Pharmacol Pharmacother 2014;5:203-5.
34 Wasserman DA, Weinstein MG, Havassy BE, et al. Factors associated with lapses to heroin use during methadone maintenance. Drug Alcohol Depend 1998;52:183-92.

35 Balhara YPS. Time to include buprenorphine-naloxone combination in the who model list of essential medicines. J Opioid Manag 2013;9:237.

36 Fitzcharles M-A, Baerwald C, Ablin J, et al. Efficacy, tolerability and safety of cannabinoids in chronic pain associated with rheumatic diseases (fibromyalgia syndrome, back pain, osteoarthritis, rheumatoid arthritis): a systematic review of randomized controlled trials. Schmerz 2016;30:47-61.

37 Lynch ME, Ware MA. Cannabinoids for the treatment of chronic noncancer pain: an updated systematic review of randomized controlled trials. J Neuroimmune Pharmacol 2015;10:293-301.

38 Martín-Sánchez E, Furukawa TA, Taylor J, et al. Systematic review and meta-analysis of cannabis treatment for chronic pain. Pain Med 2009;10:1353-68.

39 Whiting PF, Wolff RF, Deshpande S, et al. Cannabinoids for medical use: a systematic review and meta-analysis. JAMA 2015;313:2456-73.

40 Gates PJ, Albertella L, Copeland J. The effects of cannabinoid administration on sleep: a systematic review of human studies. Sleep Med Rev 2014:18:477-87.

41 Walsh Z, Gonzalez R, Crosby K, et al. Medical cannabis and mental health: a guided systematic review. Clin Psychol Rev 2017;51:15-29.

42 MacCallum CA, Russo EB. Practical considerations in medical cannabis administration and dosing. Eur J Intern Med 2018;49:12-19.

43 Khaiser MPM, Ahrari S, Pasetka M, et al. Medical cannabis dosing strategies in pain-related conditions: a scoping review of current literature. J Pain Manage 2016;9:449-63.

44 Allan GM, Ramii J, Perry D, et al. Simplified guideline for prescribing medical cannabinoids in primary care. Can Fam Physician 2018;64:111-20.

45 Busse JW, Craigie S, Juurlink DN, et al. Guideline for opioid therapy and chronic noncancer pain. CMAJ 2017;189:E659-66.

46 Bloom HG, Ahmed I, Alessi CA, et al. Evidence-Based recommendations for the assessment and management of sleep disorders in older persons. J Am Geriatr Soc 2009;57:761-89.

47 López-Pelayo H, Batalla A, Balcells MM, et al. Assessment of cannabis use disorders: a systematic review of screening and diagnostic instruments. Psychol Med 2015;45:1121-33.

48 Saunders JB, Aasland OG, Babor TF, et al. Development of the Alcohol Use Disorders Identification Test (AUDIT): WHO Collaborative Project on Early Detection of Persons with Harmful Alcohol Consumption--II. Addiction 1993;88:791-804.

49 Grant BF, Dawson DA, Stinson FS, et al. The alcohol use disorder and associated disabilities interview Schedule-IV (AUDADIS-IV): reliability of alcohol consumption, tobacco use, family history of depression and psychiatric diagnostic modules in a general population sample. Drug Alcohol Depend 2003;71:7-16.

50 Butler SF, Budman SH, Fernandez KC, et al. Development and validation of the current opioid misuse measure. Pain 2007;130:144-56.

51 Lake S, Kerr T, Montaner J. Prescribing medical cannabis in Canada: are we being too cautious? Can J Public Health 2015;106:e328-30.

52 Hayashi K, Milloy M-J, Lysyshyn M, et al. Substance use patterns associated with recent exposure to fentanyl among people who inject drugs in Vancouver, Canada: a cross-sectional urine toxicology screening study. Drug Alcohol Depend 2018;183:1-6.

53 Adamson SJ, Kay-Lambkin FJ, Baker AL, et al. An improved brief measure of cannabis misuse: the cannabis use disorders identification Test-Revised (CUDIT-R). Drug Alcohol Depend 2010;110:137-43.

54 Moher D, Shamseer L, Clarke M, et al. Preferred reporting items for systematic review and meta-analysis protocols (PRISMA-P) 2015 statement. Syst Rev 2015;4:1.

55 Moher D, Liberati A, Tetzlaff J, et al. Preferred reporting items for systematic reviews and meta-analyses: the PRISMA statement. PLOS Med 2009;6:e1000097.

56 Roen $\mathrm{K}$, Arai L, Roberts $\mathrm{H}$, et al. Extending systematic reviews to include evidence on implementation: methodological work on a review of community-based initiatives to prevent injuries. Soc Sci Med 2006:63:1060-71

57 Armstrong R, Waters $\mathrm{E}$, Roberts $\mathrm{H}$, et al. The role and theoretical evolution of knowledge translation and exchange in public health. $J$ Public Health 2006;28:384-9.

58 Atkins D, Best D, Briss PA, et al. Grading quality of evidence and strength of recommendations. BMJ 2004;328:1490. 
59 Guyatt GH, Oxman AD, Vist GE, et al. Grade: an emerging consensus on rating quality of evidence and strength of recommendations. $B M J$ 2008;336:924-6.

60 Guyatt GH, Oxman AD, Kunz R, et al. What is "quality of evidence" and why is it important to clinicians? BMJ 2008;336:995-8.

61 Guyatt GH, Oxman AD, Kunz R, et al. Going from evidence to recommendations. BMJ 2008;336:1049-51.

62 Jaeschke R, Guyatt GH, Dellinger P, et al. Use of grade grid to reach decisions on clinical practice guidelines when consensus is elusive. BMJ 2008;337:a744.
63 Schünemann HJ, Schünemann AHJ, Oxman AD, Brozek J, et al. Grading quality of evidence and strength of recommendations for diagnostic tests and strategies. $B M J$ 2008;336:1106-10.

64 Guyatt GH, Oxman AD, Kunz R, et al. Incorporating considerations of resources use into grading recommendations. $B M J$ 2008;336:1170-3.

65 National Institutes of Health (NIH). Available: https://www.nhlbi. nih.gov/health-topics/study-quality-assessment-toolsAccessed [Accessed 21 Sep 2019]. 